\title{
Relation between color-deconfinement and chiral restoration
}

\author{
Kenji Fukushima * a

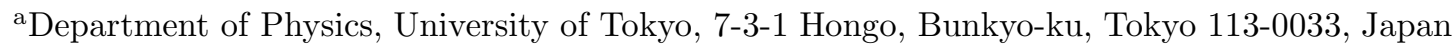

\begin{abstract}
We discuss the relation between the Polyakov loop and the chiral order parameter at finite temperature by using an effective model. We clarify why and how the pseudo-critical temperature associated with the Polyakov loop should coincide with that of the chiral condensate.
\end{abstract}

Quantum Chromodynamics (QCD) is believed to undergo phase transitions from hadronic matter to a quark-gluon plasma (QGP) at high temperature. The QGP phase transitions can consist of two distinct transitions - the deconfinement transition and the chiral phase transition. Several theoretical studies [1] have clarified that confinement would be closely related to chiral dynamics and the confined phase should be accompanied by spontaneous breaking of the chiral symmetry. This means that the deconfinement transition temperature must be lower than the chiral phase transition temperature.

Actually, in the presence of massive fundamental quarks, neither the deconfinement transition nor the chiral phase transition has any welldefined order parameter. In the lattice QCD simulations [2], it has been observed that the approximate order parameters for two phase transitions, that is, the Polyakov loop and the chiral condensate both show crossover behavior like the magnetization under an external magnetic field. We cannot define the critical temperature any more. Instead, we can only locate the "critical point" by the peak of susceptibility, namely the pseudocritical temperature. The lattice QCD simulations have revealed that the respective peaks of the Polyakov loop and the chiral susceptibilities are located at the same temperature. It has been a puzzle why and how those pseudo-critical temperatures should be identical.

\footnotetext{
*We acknoledge the support of the Japan Society for the Promotion of Science for Young Scientists. The present address of the author is: Center for Theoretical Physics, Massachusetts Institute of Technology, Cambridge, MA.
}

A simple explanation has been proposed by Satz [3. At low temperature with spontaneous chiral symmetry breaking, the center symmetry breaking is suppressed due to constituent quark mass. The Polyakov loop is small as a result. In the chiral symmetric phase at high temperature, the Polyakov loop can become large. Therefore the constituent quark mass should govern the Polyakov loop behavior rather than deconfinement would. In fact, Gocksch and Ogilvie 4] had constructed an effective model based on the strong coupling expansion and argued that Satz's argument is embodied qualitatively. Recently it has been shown by the present author [5] that the Gocksch-Ogilvie model works pretty well and Satz's argument makes too much of the chiral phase transition. The Polyakov loop dynamics is also important to understand the coincidence of pseudo-critical temperatures.

The Gocksch-Ogilvie model is given by the effective action 45

$$
\begin{aligned}
& S_{\text {eff }}^{(r)}[L, \lambda]=-\mathrm{e}^{-\sigma a / T} \sum_{\text {n.n. }} \operatorname{Tr}_{\mathrm{c}} L(\vec{n}) \operatorname{Tr}_{\mathrm{c}} L^{\dagger}(\vec{m}) \\
& +\frac{\operatorname{dim}(r)}{2} \sum_{m, n} \lambda(n) V(n, m) \lambda(m) \\
& -\frac{N_{\mathrm{f}}}{4} \sum_{\vec{n}} \operatorname{Tr}_{\mathrm{c}} \ln \left[\cosh \left(N_{\tau} E\right)+\frac{1}{2}\left(L^{(r)}+L^{(r) \dagger}\right)\right],
\end{aligned}
$$

where $L(\vec{n})$ is the Polyakov loop in the fundamental representation defined on the lattice by

$L(\vec{n})=\prod_{n_{d}=a}^{N_{\tau} a} U_{d}\left(\vec{n}, n_{d}\right)$, 
in the $d$ dimensional space-time. $r$ is the representation of dynamical quarks. The Polyakov loop in the adjoint representation $(r=\operatorname{adj})$ can be expressed in terms of the fundamental one as

$L_{a b}^{(\operatorname{adj})}=2 \operatorname{Tr}_{\mathrm{c}}\left[t_{a} L t_{b} L^{\dagger}\right]$

where the matrices $t_{a}$ form a fundamental representation of the $\mathrm{SU}\left(N_{\mathrm{c}}\right)$ group. $\lambda(n)$ is the meson field, $\operatorname{Tr}_{\mathrm{c}}$ the trace with respect to the color indices, and $N_{\mathrm{f}}$ the number of flavors which is fixed as $N_{\mathrm{f}}=2$ in the present analyses. $\operatorname{dim}(r)$ is the dimension of the $r$ representation, that is, $\operatorname{dim}($ fund $)=N_{\mathrm{c}}$ and $\operatorname{dim}(\operatorname{adj})=N_{\mathrm{c}}^{2}-1$. The meson hopping propagator $V(n, m)$ and the quasiquark energy (constituent quark mass) $E$ are defined respectively as

$V(n, m)=\frac{1}{2(d-1)} \sum_{\hat{j}}\left(\delta_{n, m+\hat{j}}+\delta_{n, m-\hat{j}}\right)$,

$E=\sinh ^{-1}\left(\sqrt{\frac{d-1}{2} \lambda}+m_{q}\right)$

with $\hat{j}$ running from 1 to $d-1$ only in the spatial directions. $\sigma$ in Eq. (1) is the string tension fixed as $\sigma=(425 \mathrm{MeV})^{2}$. The lattice spacing $a$ and the current quark mass $m_{q}$ are treated as the model parameters.

We shall adopt the mean field (Weiss) approximation for the Polyakov loop $L$. In this approximation, we take account of the fluctuation of the individual Polyakov loop surrounded by a constant mean field. As for the chiral order parameter, $\lambda$ is simply treated as a constant mean field. Detailed calculations are given in Ref. [5].

In Table 1 we list the values of the model parameters and several resulting outputs for fundamental quarks. $T_{\mathrm{d}}$ is the deconfinement temperature estimated from the gluonic contribution

\begin{tabular}{c|cccc}
\hline \hline & $a^{-1}$ & $m_{q}$ & $T_{\mathrm{d}}$ & $T_{\mathrm{c}}$ \\
\hline $\mathrm{I}$ & $432 \mathrm{MeV}$ & $5.7 \mathrm{MeV}$ & $208 \mathrm{MeV}$ & $\sim 187 \mathrm{MeV}$ \\
\hline II & $333 \mathrm{MeV}$ & $7.4 \mathrm{MeV}$ & $270 \mathrm{MeV}$ & $\sim 230 \mathrm{MeV}$ \\
\hline \hline
\end{tabular}

Table 1

Model parameters and resulting outputs for fundamental quarks. alone and $T_{\mathrm{c}}$ is the pseudo-critical temperature defined by the peak of susceptibility. Parameter I is determined to fit the pion mass and the $\rho$ meson mass, while Parameter II reproduces the pion mass and the empirical value of the deconfinement temperature, i.e., $T_{\mathrm{d}}=270 \mathrm{MeV}$. These two parameter sets happen to correspond to two typical cases, as explained below.
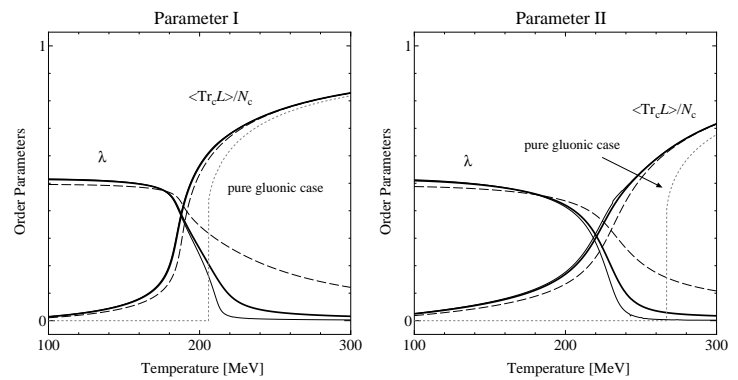

Figure 1. Order parameters: the thick solid curve for $m_{q}=5.7 \mathrm{MeV}$ in Parameter $\mathrm{I}$ and $m_{q}=7.4 \mathrm{MeV}$ in Parameter II, the thin solid and dashed curves for $m_{q}=1 \mathrm{MeV}$ and $50 \mathrm{MeV}$ respectively.

The numerical results are shown in Fig. 1 together with the results for several different $m_{q}$. In both cases we can see the order parameters for deconfinement and chiral restoration indicating crossover behavior simultaneously. Although the results in Fig. 1]just seem to support Satz's argument, the relation between color-deconfinement and chiral restoration has richer physical contents, as inferred from Fig. 2

For $m_{q}=1 \mathrm{MeV}$ with Parameter I, two peaks appear clearly in the behavior of slope, $\chi_{\mathrm{t}}^{\lambda}=$ $-\partial \lambda / \partial T$. One peak around $T_{\mathrm{c}} \simeq 187 \mathrm{MeV}$ corresponds to the remnant of the first order deconfinement transition. The other around $T \simeq 210 \mathrm{MeV}$ stems from the second order chiral phase transition, which agrees with the thin curve in Fig. 1 Therefore the coincidence of the pseudo-critical temperatures signifies not the chiral phase transition but the deconfinement transition. In this sense, we can say that the Deconfinement Dominance is realized in the simultaneous crossovers.

The Deconfinement Dominance is seen in $\mathrm{Pa}$ - 

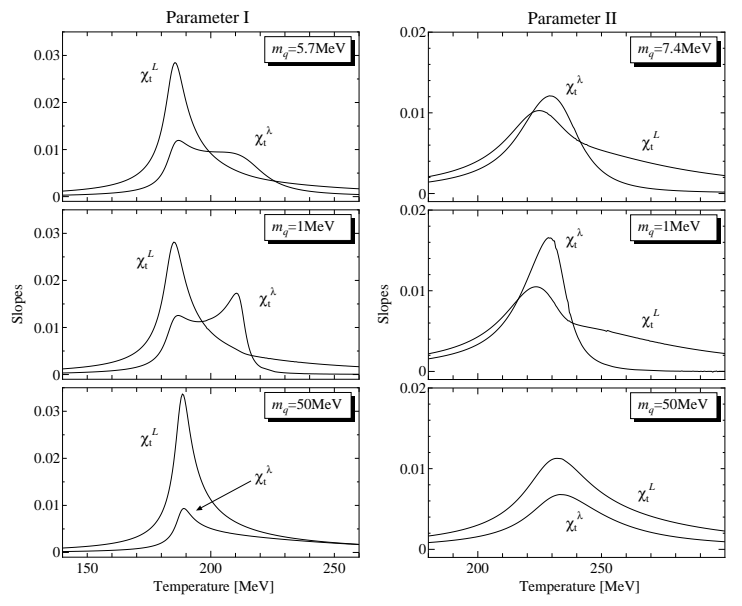

Figure 2. Temperature slopes

rameter I because $T_{\mathrm{d}}$ is too low in this case. Then, can we expect the Chiral Dominance in Parameter II? If so, it would be an ideal realization of Satz's argument. The answer is, however, rather profound in fact.

For $m_{q}=1 \mathrm{MeV}$ with Parameter II, there appears only one peak around $T_{\mathrm{c}} \simeq 225 \mathrm{MeV}$. This peak results from the second order chiral phase transition, which agrees with the thin curve in Fig.1 Thus the coincidence of the pseudo-critical temperatures in this case can be understood according to Satz's argument. Nevertheless this is not the Chiral Dominance because of the theoretical requirement that chiral restoration occurs at higher temperature than color-deconfinement does. We can prove that the Gocksch-Ogilvie model satisfies this requirement [5]. We can say that chiral restoration would be blocked by the Polyakov loop behavior. Therefore, the simultaneous crossovers are really simultaneous. In other words, we have only one soft-mode associated with the susceptibility peaks [ 6 . We would emphasize that this understanding is novel and non-trivial.

Finally, we shall present the results for adjoint quarks $(r=\operatorname{adj})$ with Parameter I in Fig. 3 In this case, the center symmetry is not broken explicitly. The results certainly show a clear first order transition in the Polyakov loop and agree qualitatively with the lattice aQCD results [7.

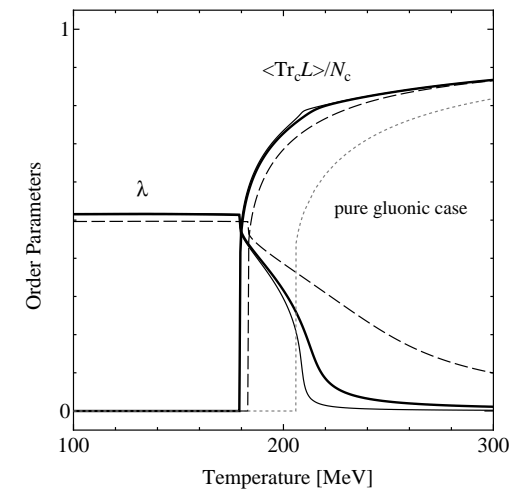

Figure 3. Order parameters for adjoint quarks with Parameter I.

In summary, the Gocksch-Ogilvie model can give a nice guideline to get a deeper insight into the relation between the Polyakov loop dynamics and the chiral dynamics. The underlying physics of simultaneous crossovers is to be investigated more seriously in the future.

\section{REFERENCES}

1. A. Casher, Phys. Lett. B83 (1979) 395.

G. 't Hooft, in: Recent Developments in Gauge Theories, Eds. G. 't Hooft et al., (Plenum, New York, 1980).

2. F. Karsch and E. Laermann, Phys. Rev. D50 (1994) 6954.

S. Aoki, M. Fukugita, S. Hashimoto, N. Ishizuka, Y. Iwasaki, K. Kanaya, K. Kuramashi, H. Mino, M. Okawa, A. Ukawa, and T. Yoshié, Phys. Rev. D57 (1998) 3910.

3. H. Satz, Nucl. Phys. A642 (1998) 130.

S. Digal, E. Laermann, and H. Satz, Eur. Phys. J. C18 (2001) 583.

4. A. Gocksch and M. Ogilvie, Phys. Rev. D31 (1985) 877.

5. K. Fukushima, Phys. Lett. B553 (2003) 38; Phys. Rev. D68 (2003) 045004.

6. Y. Hatta and K. Fukushima, e-print: hep-ph/0307068.

7. F. Karsch and M. Lütgemeier, Nucl. Phys. B550 (1999) 449. 